\title{
The liver and pancreas in acute necrotising pancreatitis
}

\author{
W. K. BLENKINSOPP \\ From the Department of Histopathology, St Mary's Hospital, London W2, UK
}

SUMMaRY Pancreatic fibrosis was found in 23 of 31 cases of acute necrotising and haemorrhagic pancreatitis; however, in the cases with a history of six days or less neither the extent nor the frequency of fibrosis differed significantly from those in controls. There was no histological evidence that duct or vascular lesions are necessary for the disease to occur. The liver was examined in 26 cases and showed cholestasis in 12, including 10 of 20 cases without a biliary aetiology and only two of six cases with biliary tract disease. In no case did the liver show specific features of alcoholic damage.

In the Bristol area during the period 1950 to 1969 , 590 cases of acute pancreatitis were identified (Trapnell and Duncan, 1975); biliary tract disease was present in $54 \%$, chronic alcoholism in $4 \%$, and $34 \%$ were idiopathic. An increasing proportion of cases due to alcoholism occurred during the 20 years, and alcohol was considered to be the cause in $26 \%$ of 78 patients studied in Glasgow in 1971-72 (Imrie and Whyte, 1975); in this series $51 \%$ of patients had biliary disease, and only $13 \%$ were considered idiopathic.

The liver was therefore examined in a series of cases of acute pancreatitis to see if biliary disease was manifested by histological evidence of large bile duct obstruction, and if evidence of alcoholic damage to the liver was present. The pancreas and adjacent fat were examined to establish the diagnosis and to identify any other abnormalities.

\section{Material and methods}

Twenty-six cases of acute necrotising haemorrhagic pancreatitis were identified from the necropsy reports of the period 1963-77, and a further five cases were found among the surgical material of the last three years following a trend towards operation in this disease. All patients were in their first attack. Biliary aetiology was diagnosed on clinical or necropsy evidence of extrahepatic biliary tract disease, and alcoholic aetiology was diagnosed on the clinical history; the remaining cases were considered idiopathic - none was associated with steroids and none followed abdominal operation. Paraffin sections of

Received for publication 29 December 1977 formalin-fixed liver, pancreas, and adjacent fat were cut at $5 \mu$ and examined after staining with haematoxylin and eosin, van Gieson, periodic acid Schiff after diastase, and for reticulin, as appropriate. In most cases one block of liver and two blocks of pancreas were examined. The pancreas was also examined in two control groups-22 diabetics and 31 non-diabetics.

\section{Results}

Three findings in the liver were considered suggestive of large duct obstruction: cholestasis, marginal duct proliferation in the portal tracts, and acute portal tract inflammation. Only eight of the 26 cases had none of these features (Table 1), and all three were present in two of the six biliary and four of the 17 idiopathic cases.

Table 1 Liver in 26 cases of acute pancreatitis

\begin{tabular}{lllll}
\hline & \multicolumn{2}{c}{ Portal trocts } & & \\
\cline { 2 - 5 } & Cholestasis & $\begin{array}{l}\text { Inflammation } \\
\text { with } \\
\text { neutrophils }\end{array}$ & $\begin{array}{l}\text { Marginal duct None } \\
\text { proliferation }\end{array}$ \\
\hline Idiopathic (17) & 8 & 10 & 7 & 5 \\
Biliary (6) & 2 & 4 & 2 & 2 \\
Alcoholic (3) & 2 & 2 & 0 & 1 \\
Total (26) & 12 & 16 & 9 & 8 \\
\hline
\end{tabular}

Marked excess fat was present in the liver in two of 17 idiopathic cases, in one of six biliary, and in none of three alcoholic cases. There were no cases with findings more strongly suggestive of alcoholic damage (hyaline, acute alcoholic hepatitis, and 
centrizonal sclerosing hyaline necrosis). Antitrypsin bodies were present in one of the 26 cases.

The findings in the pancreas are shown in Table 2. No difference was apparent between the various aetiological groups. The extent of necrosis of pancreatic tissue varied considerably from almost complete to none (in four late cases); in all cases the pancreas was surrounded by necrotic and haemorrhagic fat with an inflammatory reaction.

Table 2 Pancreas in 31 cases of acute pancreatitis

\begin{tabular}{lrrrrr}
\hline & Necrosis & Fibrosis & $\begin{array}{l}\text { Ducts } \\
\text { damaged }\end{array}$ & $\begin{array}{l}\text { Vascular } \\
\text { throm- } \\
\text { bosis }\end{array}$ & $\begin{array}{l}\text { Vessel } \\
\text { wall } \\
\text { necrosis }\end{array}$ \\
\hline Idiopathic (20) & 17 & 15 & 8 & 8 & 7 \\
Biliary (6) & 6 & 3 & 1 & 4 & 3 \\
Alcoholic (5) & 4 & 5 & 2 & 1 & 2 \\
Total (31) & 27 & 23 & 11 & 13 & 12 \\
\hline
\end{tabular}

Patchy fibrosis was present in the pancreas in 23 of the 31 cases. Fourteen cases were of more than six days' duration, and fibrosis was present in 12 of these; the fibrosis probably occurred during the current illness. Seventeen cases had a history of six days or less, and fibrosis was present in 11 of these; one was clinically of alcoholic aetiology, and the fibrosis in this case could be attributed to alcohol; of the remaining 10 cases with fibrosis, this was active and recent in three, and despite the short history this had presumably occurred during the current illness. The remaining seven cases of old fibrosis in 13 early cases were compared with the two control groups, in which old fibrosis was found in the pancreas of 11 of 22 diabetics and of 10 of 31 nondiabetics. In the non-diabetic group two cases with fibrosis had a history of high alcohol consumption, and these were excluded from the comparison; the frequency of old fibrosis did not differ significantly between the early pancreatitis, diabetic, and nondiabetic groups ( $\chi^{2}$ test), nor was there a recognisable difference in extent of fibrosis.

The pancreatic ducts were plugged by inspissated secretion and showed mild inflammatory changes in 11 of the 31 cases; no dilated ducts were found. Similar lesions were found in 11 of the 53 control cases; the difference between pancreatitis and control cases was not statistically significant. In the controls there was a significant $(P<0.005)$ association between duct plugging and fibrosis; 30 cases had neither, nine both, 12 fibrosis only, and two plugs only. This relationship was not found in the early cases of pancreatitis.

Vascular lesions (thrombosis and/or fibrinoid necrosis) were found in the small vessels of the pancreas in 18 of the 31 cases; similar lesions were not found in the control groups.

\section{Discussion}

All the patients were in their first clinical attack of acute pancreatitis, and the finding of fibrosis in more than half the early cases was unexpected. However, the fibrosis was present in scanty small foci and did not differ quantitatively from that in some of the control cases, and the difference in frequency of fibrosis between the early pancreatitis group and the controls was not statistically significant. Kreel and Sandin (1973) found that duct diameter increased with age over 50 years, but this was not associated with fibrosis or chronic inflammation; in the present series the frequency of fibrosis was not related to age in any group.

The variation in extent of necrosis of pancreatic tissue was anticipated, as most pathologists have on occasion cut through a mass of haemorrhagic and necrotic fat to find the pancreas relatively undamaged within, and Maclean (1977) has reported three cases at four to 14 weeks after onset in which the comparative normality of the residual pancreas was a striking finding.

Experimentally, pancreatic duct obstruction produces oedema without necrosis and haemorrhage, whereas the addition of vascular occlusion produces both these features and a much higher mortality (Goodhead, 1969). Eleven of the 31 cases in the present series had pancreatic duct plugging by inspissated secretion and some inflammation, but none was dilated. Duct plugging was also present in a similar proportion of the controls, and was significantly associated with small foci of fibrosis; this relationship may be causal, but neither the plugging nor the fibrosis appear to play a significant role in the production of acute pancreatitis.

Eighteen cases had small vessel lesions (thrombosis and/or fibrinoid necrosis of the wall), mainly involving venules, but these appeared closely related to adjacent necrosis and were thus probably secondary. There was no evidence of generalised small vessel lesions in other organs, and these lesions were not found in the pancreas in the control groups.

In 116 patients with acute pancreatitis, Storck et al. (1976) found $75 \%$ of the patients had hyperbilirubinaemia and about half of these had a raised alkaline phosphatase, suggesting that the cause was cholestasis. Forty-three per cent of the cases were associated with gallstones, and in this group hyperbilirubinaemia and raised alkaline phosphatase were found in two-thirds. The present study confirmed that cholestasis is common in acute pancreatitis and not clearly related to a biliary tract aetiology; it was 
present in 10 of 20 non-biliary cases and in only two of six biliary cases. Other features suggestive of large duct obstruction were also present in both biliary and non-biliary cases (Table 1), and it is clear that they can occur singly or in combination in patients without large bile duct obstruction.

The absence of specific evidence of alcoholic damage in the liver suggests that liver histology is not a satisfactory way of diagnosing an alcoholic aetiology in acute pancreatitis, though the number of alcoholic cases is too small for a firm conclusion. Storck et al. (1976) described macroscopic steatosis of the liver in $62 \%$ of the alcoholic cases and in about $25 \%$ of the non-alcoholic cases in their series of 116 patients with acute pancreatitis, but did not comment on the histology of the liver.

Trypsin probably plays some part in the development of acute pancreatitis (Glazer, 1975; HermonTaylor, 1977), and Novis et al. (1975) found a significant excess of heterozygous (PiMZ) $\alpha_{1}$-antitrypsin deficient subjects among 110 cases of chronic pancreatitis. In the 26 livers examined, only one had antitrypsin bodies, which is the expected incidence in the general population (Blenkinsopp and Haffenden, 1977).

Glazer (1975) has reviewed the various theories of pathogenesis of acute pancreatitis, including McCutcheon's (1968) proposal of reflux of activated duodenal enzymes. The present study provides no evidence for or against this theory but supports it to the extent that neither duct obstruction nor vascular occlusion appears to be necessary.

I am grateful to Dr Stephanie Wilson for permission to incorporate seven cases in this study.

\section{References}

Blenkinsopp, W. K., and Haffenden, G. P. (1977). Alpha-1-antitrypsin bodies in the liver. Journal of Clinical Pathology, 30, 132-137.

Glazer, G. (1975). Haemorrhagic and necrotizing pancreatitis. British Journal of Surgery, 62, 169-176.

Goodhead, B. (1969). Vascular factors in the pathogenesis of acute haemorrhagic pancreatitis. Annals of the Royal College of Surgeons, 45, 80-97.

Hermon-Taylor, J. (1977). An aetiological and therapeutic review of acute pancreatitis. British Journal of Hospital Medicine, 18, 546-552.

Imrie, C. W., and Whyte, A. S. (1975). A prospective study of acute pancreatitis. British Journal of Surgery, 62, 490-494.

Kreel, L., and Sandin, B. (1973). Changes in pancreatic morphology associated with aging. Gut, 14, 962-970.

McCutcheon, A. D. (1968). A fresh approach to the pathogenesis of pancreatitis. Gut, 9, 296-310.

Maclean, N. (1977). The role of the surviving pancreas in late fatalities of acute pancreatitis. British Journal of Surgery, 64, 345-346.

Novis, B. H., Bank, S., Young, G. O., and Marks, I. N. (1975). Chronic pancreatitis and alpha-1-antitrypsin. Lancet, 2, 748-749.

Storck, G., Pettersson, G., and Edlund, Y. (1976). A study of autopsies upon 116 patients with acute pancreatitis. Surgery, Gynecology and Obstetrics, 143, 241-245.

Trapnell, J. E., and Duncan, E. H. L. (1975). Patterns of incidence in acute pancreatitis. British Medical Journal, 2, 179-183.

Requests for reprints: to Dr W. K. Blenkinsopp, Histopathology Department, St Mary's Hospital, Praed Street, London W2 1NY. 